\title{
P38 mitogen activated protein kinase expression and regulation by interleukin-4 in human B cell non-Hodgkin lymphomas
}

\author{
Hu Ding • Ali M. Gabali • Stephen D. Jenson • \\ Megan S. Lim • Kojo S. J. Elenitoba-Johnson
}

Received: 23 July 2009 /Accepted: 30 September 2009 /Published online: 21 October 2009

(C) Springer-Verlag 2009

\begin{abstract}
The prevalence and regulation of p38 mitogen activated protein kinase (MAPK) expression in human lymphomas have not been extensively studied. In order to elucidate the role of p38 MAPK in lymphomagenesis, we examined the expression of native and phosphorylated $\mathrm{p} 38$ (p-p38) MAPK in cell lines derived from human hematopoietic neoplasms including B cell lymphoma-derived cell lines using Western blot analysis. The p-p38 MAPK protein was also analyzed in $30 \mathrm{~B}$ cell non-Hodgkin lymphoma (NHL) tissue biopsies by immunohistochemistry. Our results show that the expression of p38 MAPK was upregulated in most of the cell lines as compared with peripheral blood lymphocytes, while the expression of p-p38 MAPK was more variable. A subset of B cell NHL biopsies showed increased expression of p-p38 MAPK relative to reactive germinal center cells. Interleukin-4 (IL-4) induced a dose-dependent increase in the expression of p-p38 MAPK (1.6- to 2.8-fold) in cell lines derived from activated B cell-like diffuse large B cell lymphoma (DLBCL) but not those from germinal center-like DLBCL. No change was seen in native p38 MAPK. The in vitro
\end{abstract}

Ali M. Gabali and Hu Ding have contributed equally to this manuscript.

Electronic supplementary material The online version of this article (doi:10.1007/s12308-009-0049-5) contains supplementary material, which is available to authorized users.

A. M. Gabali • M. S. Lim • K. S. J. Elenitoba-Johnson $(\bowtie)$

Department of Pathology, University of Michigan,

4061 BSRB, 109 Zina Pitcher Place,

Ann Arbor, MI 48109, USA

e-mail: kojoelen@umich.edu

H. Ding $\cdot$ S. D. Jenson

University of Utah Health Sciences Center,

Salt Lake City, UT, USA kinase activity of p38 MAPK, however, was induced (1.6to 3.2-fold) in all five cell lines by IL-4. Quantitative fluorescent RT-PCR demonstrated that all four isoforms of p38 MAPK gene were expressed in the lymphoma cell lines, with $\mathrm{p} 38 \gamma$ and $\mathrm{p} 38 \beta$ isoforms being predominant. IL4 stimulation increased the expression of $\beta, \gamma$, and $\delta$ isoforms but not $\alpha$ isoform in two cell lines. In conclusion, there is constitutive expression and activation of p38 MAPK in a large number of B-lymphoma-derived cell lines and primary lymphoma tissues, supportive of its role in lymphomagenesis. The differential IL-4 regulation of p38 MAPK expression in cell lines derived from DLBCL may relate to the cellular origin of these neoplasms.

Keywords p38 MAPK · p-p38 · Isoforms · RT-PCR · IL-4 · In vitro kinase assay

\section{Introduction}

The p38 mitogen activated protein kinase (MAPK) family consists of serine-threonine protein kinases which are activated in response to stress, cytokine, or growth factor stimulation [1-5]. P38 MAPK plays a critical role in various signaling pathways that regulate cell proliferation, differentiation, apoptosis, and induction of cytokine production [6-12]. Recent studies have identified four isoforms of p38 MAPK in mammals, and these have been designated as $\mathrm{p} 38 \alpha$ [2], p38 $\beta[13,14], \mathrm{p} 38 \gamma[15,16]$, and p38ठ [17, 18]. Further studies have indicated that these protein kinases represent related, but significantly distinct, MAPK subgroups [19-24].

The role of p38 MAPK in the physiology of normal hematopoietic cells and the pathogenesis of lymphoproliferative disorders is largely unknown. The p38 MAPK 
pathway is necessary for CD40-induced gene expression and proliferation in normal B lymphocytes [25]. Our previous studies using cDNA microarray analysis and immunohistochemistry demonstrated that transformation of follicular lymphoma to diffuse large B cell lymphoma (DLBCL) is associated with increased expression of genes within the ras-p38 MAPK pathway and upregulation of phosphorylated p38 MAPK protein [26].

To investigate the role of p38 MAPK in lymphomagenesis, we analyzed the prevalence and pattern of expression of p38 MAPK in a diverse group of human lymphoma cell lines and tissue biopsies from B cell nonHodgkin lymphoma (NHL). Secondly, we examined the effects of interleukin-4 (IL-4) on gene expression and in vitro activity of $\mathrm{p} 38$ MAPK in five cell lines derived from DLBCL. Our studies show constitutive expression of $\mathrm{p} 38$ MAPK and its activated form in cell lines derived from human lymphoid tumors as well as in primary tissue biopsy samples. Furthermore, there is differential expression and regulation of p38 MAPK isoforms by IL-4 in lymphoma-derived cell lines. We also show that IL-4 is a potent regulator of p38 MAPK in lymphoma-derived cell lines which may be dependent on their putative cell of origin.

\section{Materials and methods}

\section{Cell studies}

Cell lines derived from a variety of human B cell lymphoma-derived cell lines, including activated B cell (ABC) subtype (OCI-Ly-10 and Sudhl-5) and germinal center B cell (GCB) subtypes (OCI-Ly-1, Sudhl-4, and Sudhl-6) of DLBCL-derived cell lines (Table 1), were obtained from either American Type Culture Collection (Rockville, MD) and German Collection of Microorganism and Culture (Braunschweig, Germany). OCI-Ly-1, Sudhl-4, and Sudhl-6 carry the $\mathrm{t}(14 ; 18)$ translocation but OCI-Ly10 and Sudhl-5 do not [27]. The cells were grown to subconfluence before individual studies in RPM1-1640 medium (Nova-Tech, Inc.) supplemented with 10\% fetal bovine serum (FBS) and antibiotics. In IL-4 stimulation studies, the cells were incubated in FBS-free RPM1-1640 media for $24 \mathrm{~h}$ at a concentration of $4 \times 10^{5}$ cells $/ \mathrm{ml}$, followed by incubation in fresh FBS-free RPM1-1640 media supplemented with IL-4 of concentrations ranging from 5 to $30 \mathrm{ng} / \mathrm{ml}$ for $24 \mathrm{~h}$. Preliminary experiments have confirmed that incubating cell lines with IL-4 at the designated concentration for $24 \mathrm{~h}$ did not alter cell viability in which more than $95 \%$ of the cells were viable as determined by staining with Trypan blue. After 24-h treatment with IL-4, the cells were collected by centrifugation at $4,000 \mathrm{rpm}$ at room temperature for $10 \mathrm{~min}$, followed by two washes with ice-cold PBS. The resultant cell pellets were stored at $-80^{\circ} \mathrm{C}$ for further analyses.

Preparation of cell lysate for in vitro kinase assays and Western blotting analyses

The cell pellets were thawed on ice and resuspended in icecold cell lysis buffer (20 mM Tris (pH 7.5), $150 \mathrm{mM} \mathrm{NaCl}$, $1 \mathrm{mM}$ EDTA, $1 \mathrm{mM}$ EGTA, 1\% Triton X-100, $2.5 \mathrm{mM}$ sodium pyrophosphate, $1 \mathrm{mM} \mathrm{Na} \mathrm{VO}_{4}, 1 \mu \mathrm{g} / \mathrm{ml}$ leupeptin, and $1 \mathrm{mM} \mathrm{PMSF}$ ) at a concentration of $2 \times 10^{7}$ cells $/ \mathrm{ml}$. After incubation on ice for $5 \mathrm{~min}$, the mixture was sonicated four times of $5 \mathrm{~s}$ each. The cell lysate was then centrifuged at $14,000 \mathrm{rpm}$ at $4^{\circ} \mathrm{C}$ for $10 \mathrm{~min}$, and the supernatant was collected. After the protein concentration of the supernatant was measured with BCA assay (Pierce
Table 1 B cell lymphomaderived cell lines used for Western blot analysis

$B$ B cell, $A L L$ acute lymphoblastic leukemia, $B L$ Burkitt lymphoma, $E B V$ Epstein Barr virus transformed, $M C L$ mantle cell lymphoma, $P E L$ pleural effusion-based lymphoma, $N H L$ non-Hodgkin lymphoma

\begin{tabular}{llllll}
\hline Name & Lineage & Type & Name & Lineage & Type \\
\hline NCEB & B cell & MCL/t $(11 ; 14)$ & OCI-Ly-2 & B cell & B-NHL \\
OCI-Ly-1 & B cell & B-NHL/t(14;18) & OCI-Ly-8 & B cell & B-NHL \\
GM607 & B cell & EBV & OCI-Ly-10 & B cell & B-NHL \\
KARPAS 422 & B cell & B-NHL/t $(14 ; 18)$ & BC-1 & B cell & PEL + AIDS \\
Raji & B cell & African BL/t $(8 ; 14)$ & BC-3 & B cell & non-AIDS PEL \\
GM697 & B cell & B-ALL/t(1;19) & Sudhl-10 & B cell & B-NHL \\
NALM-6 & B cell & B-ALL & REH & B cell & B-ALL/t(12;21) \\
GRANTA 519 & B cell & MCL/t $(11 ; 14)$ & Sudhl-8 & B cell & B-NHL \\
Sudhl-4 & B cell & B-NHL/t(14;18) & Sudhl-9 & B cell & B-NHL \\
Sudhl-5 & B cell & B-NHL & Sudhl-16 & B cell & B-NHL/t(14;18) \\
Sudhl-6 & B cell & B-NHL/t(14;18) & & & \\
Sudhl-7 & B cell & B-NHL & & & \\
OCI-Ly-7 & B cell & B-NHL & & & \\
\hline
\end{tabular}


Biotechnology, Rockford, IL), the cell lysates were stored at $-80^{\circ} \mathrm{C}$ until future use.

Western blot analysis of p38 MAPK and phospho-p38 MAPK

Aliquots $(30 \mu \mathrm{g})$ of the cell lysate proteins were separated by $12 \%$ SDS-PAGE. A positive control was established by stimulating OCI-Ly-1 cells with $25 \mu \mathrm{g} / \mathrm{ml}$ anisomycin (Sigma, St. Louis, MO) for $20 \mathrm{~min}$ at standard culture condition. Ten micrograms of the cell lysate protein of the positive control was loaded to each gel. After electrophoresis, the proteins were transferred to nitrocellulose membrane at $10 \mathrm{~V}$ for $35 \mathrm{~min}$ using a Bio-Rad semi-dry transfer cell (Bio-Rad Laboratories, Inc., Hercules, CA). The membrane was washed for $5 \mathrm{~min}$ in Tris Buffer Saline Tween (TBST) buffer followed by incubation in blocking buffer (TBST buffer with $0.5 \%$ dry milk) for $1 \mathrm{~h}$ at room temperature. The membrane was then washed three times with TBST and subsequently treated with primary antibody (Cell Signaling Technology, Beverly, MA) at 1:1,000 dilutions in antibody buffer (TBST with $0.5 \%$ BSA), overnight at $4^{\circ} \mathrm{C}$. The membrane was then washed three times in TBST buffer followed by incubation in horseradish peroxide-conjugated secondary antibody at 1:1,000 dilutions in blocking buffer, for $1 \mathrm{~h}$ at room temperature. After another three washes with TBST buffer, the blot was developed with luminol reagent (Santa Cruz Biotechnology, Santa Cruz, CA) for 1-3 min followed by exposure to Kodak Biomax Light Film (Fisher Scientific, Pittsburgh, PA). Assays were performed in triplicate for each sample and anti- $\beta$-actin antibody was used as a loading control. The density of the bands representing p38 MAPK and p-p38 MAPK was corrected by that of the positive control and designated as standardized densitometry units (SDU). In IL-4 doseresponse studies, SDU of the treated cells was corrected by the SDU of the untreated cells and designated as relative p38 MAPK and phospho-p38 MAPK expression. Mean values of the triplicate assays are reported in the results section.

In vitro p38 MAPK activity assay

In vitro p38 MAPK assay was performed using a commercial kit from Cell Signaling Technology (Beverly, MA). Briefly, $200 \mu \mathrm{g}$ of cell lysate protein was incubated with $20 \mu \mathrm{l}$ of immobilized phosphorylated p38 MAPK (p-p38 MAPK) monoclonal antibody overnight at $4^{\circ} \mathrm{C}$ in cell lysis buffer to precipitate p-p38 MAPK protein. After a wash with ice-cold cell lysis buffer and a subsequent two washes with kinase assay buffer $(25 \mathrm{mM}$ Tris $(\mathrm{pH} 7.5)$, $5 \mathrm{mM} \beta$-glycerophosphate, $2 \mathrm{mM}$ DTT, $0.1 \mathrm{mM} \mathrm{Na} 3 \mathrm{VO}_{4}$, $10 \mathrm{mM} \mathrm{MgCl} 2$, and $200 \mu \mathrm{M} \mathrm{ATP}$ ), the precipitated p-p38 MAPK was incubated overnight at room temperature, in
$50 \mu \mathrm{l}$ of kinase buffer supplemented with $2 \mu \mathrm{g}$ of the substrate protein, ATF-2 fusion protein. The reaction was terminated by adding six times the SDS sample loading buffer to the assay mixture followed by boiling the specimen for $4 \mathrm{~min}$. Aliquot of $30 \mu \mathrm{l}$ of the mixture was loaded onto and separated on 12\% SDS-PAGE gel and then transferred to nitrocellulose membrane. The phosphorylated ATF-2 protein was detected by Western blot analysis, as described above. To standardize the loading consistency, $15-\mu 1$ aliquot of the reaction mixture was loaded onto a parallel $12 \%$ SDS-PAGE gel, and after electrophoresis and transfer, total (phosphorylated and unphosphorylated) ATF-2 protein was determined by Western blot analysis. Ten micrograms of the cell lysate of the anisomycinstimulated OCI-Ly-1 cells was used as positive control for each in vitro p38 MAPK assay and subjected to the same immunoprecipitation, in vitro kinase assay, and Western blotting analysis, serving to normalize interblot variation (see statistical analysis). Ten microliters of cell extract from unstimulated NIH/3 T3 cell (Cell Signaling Technology) was used as a negative control for each assay.

Three independent experiments were performed for the in vitro p38 MAPK activity assay. The ratio of the density of phosphorylated-ATF-2 (p-ATF-2) to that of total ATF-2 was designated as the in vitro p38 MAPK activity. We normalized the in vitro $\mathrm{p} 38$ MAPK activity by dividing the in vitro $\mathrm{p} 38$ MAPK activity of individual cell lines by the in vitro p38 MAPK activity of the positive control. In IL-4 dose-response studies, we normalized the in vitro $\mathrm{p} 38$ MAPK by dividing the in vitro 338 MAPK activity of the treated cells with that of the untreated cells and expressed as relative p38 MAPK activity. The mean of the relative in vitro p38 MAPK activity of the triplicate assays are reported in the Results section.

\section{Immunohistochemistry}

Immunohistochemical studies were performed on 5- $\mu \mathrm{m}$ thick formalin-fixed paraffin-embedded tissue sections. Heatinduced epitope retrieval in citrate buffer ( $\mathrm{pH}$ 6.0) for $15 \mathrm{~min}$ was used. An avidin-biotin peroxidase complex method was employed using an automated immunostainer (Ventana Medical Systems, Tucson, Arizona). Anti-human p-p38 MAPK antibody (Cell Signaling Technology, Inc., Beverly, MA) was utilized at a dilution of 1:60. Reactive lymph nodes were used as tissue control in which germinal center B cells show negligible expression of p-p38 MAPK while interfollicular $\mathrm{T}$ cells show predominantly nuclear expression of p-p38 MAPK. Immunohistochemical analysis of p-p38 MAPK expression in lymphoma tissue samples was interpreted by two hematopathologists (M.S.L. and K.E.J.). To increase the interobserver agreement level, immunoreactivity was interpreted as positive if greater than $50 \%$ of the tumor cells showed nuclear expression of p-p38MAPK (Table 2). 
Table 2 Immunohistochemical analysis of p-p38 MAPK expression in B cell NHL tissue biopsies

\begin{tabular}{lllll}
\hline Diagnosis & $\begin{array}{l}\text { Number } \\
\text { of cases } \\
\text { analyzed }\end{array}$ & $\begin{array}{l}\text { Number } \\
\text { of cases } \\
\text { showing } \\
<50 \% \text { p-p38 }\end{array}$ & $\begin{array}{l}\text { Number } \\
\text { of cases } \\
\text { showing } \\
>50 \% \text { p-p38 }\end{array}$ & $\begin{array}{l}\text { Percentage of } \\
\text { cases showing } \\
>50 \% \text { p-p38 } \\
(\%)\end{array}$ \\
\hline CLL/SLL & 6 & 4 & 2 & 33 \\
FCL & 6 & 5 & 1 & 17 \\
MCL & 14 & 3 & 11 & 78 \\
DLBCL & 14 & 1 & 13 & 93
\end{tabular}

CLL/SLL chronic lymphocytic leukemia/small lymphocytic lymphoma, $F C L$ follicular lymphoma, $M C L$ mantle cell lymphoma, $D L B C L$ diffuse large B cell lymphoma

Quantitative fluorescence RT-PCR of isoforms of p38 MAPK

Total RNA was extracted from four DLBCL-derived cell lines (Sudhl-4, Sudhl-6, OCI-Ly-1, and OCI-Ly-10) that were treated with and without $5 \mathrm{ng} / \mathrm{ml}$ of IL-4 for $24 \mathrm{~h}$ and from phenotypically purified germinal center B-cells [28] using a modified TRIzol extraction protocol (Invitrogen, Carlsbad, CA). cDNA was reverse-transcribed using the SuperScript II kit according to manufacturer's instructions (Invitrogen, Carlsbad, CA). Quantitative fluorescent reverse transcriptasepolymerase chain reaction (RT-PCR) was performed using the LightCycler ${ }^{\mathrm{TM}}$ (Roche Molecular Biochemicals, Indianapolis, IN) and SYBR Green ITM (Molecular Probes, Eugene, OR) [29]. From each sample, $50 \mathrm{ng} / \mu \mathrm{l}$ cDNA was amplified in triplicate using primer pairs specific for each of the p38MAPK isoforms $(\alpha, \beta, \gamma$, and $\delta)$ and the control gene EMP 4.1 L1. The sequences of the primers used for quantitative RT-PCR are listed in Table 3. Reactions consisted of 45 cycles of denaturation at $94^{\circ} \mathrm{C}(0 \mathrm{~s})$, annealing at $55^{\circ} \mathrm{C}(0 \mathrm{~s})$, and extension at $72^{\circ} \mathrm{C}(15 \mathrm{~s})$. Fluorescence signals were obtained from each sample after the extension phase of each cycle. The crossing threshold or entry in to the logarithmic phase of PCR for each sample was determined by calculating the second derivative maximum using LightCycler ${ }^{\mathrm{TM}}$ software version 3.5.3.

Table 3 Primers used for quantitative RT-PCR
The expression of the $\mathrm{p} 38$ MAPK isoforms in each cell line relative to that of germinal center B cells was calculated as previously described [30]. In studies where effects of IL-4 on expression of p38 isoforms were examined, the expression values of the treated cells were further corrected by the values obtained in the untreated cells and expressed as relative expression units. The arithmetic means of the triplicate quantitative RT-PCR assays are reported in the Results section.

\section{Results}

Expression of native and phospho-p38 MAPK in human lymphoma cell lines and primary B cell non-Hodgkin lymphoma tissues

We analyzed the expression of p38 MAPK and their phosphorylation status (p-p38 MAPK) using Western blot analysis. Both the native and p-p38 MAPK proteins were detected in all B cell lymphoma cell lines (Fig. 1a, Table 1). As compared with normal lymphocytes isolated from peripheral blood, most of the cell lines derived from B cell lymphoma showed up-regulation in expression of $\mathrm{p} 38$ MAPK. However, p-p38 MAPK showed variable expression among the tested cell lines (Fig. 1a). We also evaluated the expression of p-p38 MAPK by immunohistochemistry in a panel of $30 \mathrm{~B}$ cell NHL tissue biopsies representing the most common human NHL composed of follicular lymphoma $(n=6)$; chronic lymphocytic leukemia/small lymphocytic lymphoma (CLL/SLL; $n=6$ ); mantle cell lymphoma $(n=14)$; and diffuse large B-cell lymphoma $(n=14)$. Constitutive expression of p-p38MAPK was detectable within the nucleus of lymphoma cells in $1 / 6$ (17\%) follicular lymphomas, 2/6 (33\%) CLL/SLL, 11/14 (78\%) mantle cell lymphoma (MCL), and 13/14 (93\%) DLBCLs (Fig. 1b). In contrast, reactive germinal centers from six lymph nodes showed negligible levels of p-p38 indicating that this pathway is deregulated in a variety of Blineage NHLs.

\begin{tabular}{lll}
\hline Primer & Sequence & GenBank accession number \\
\hline MAPKp38alphaF & ACTCAGATGCCGAAGATGAAC & NM_001315 \\
MAPKp38alphaR & GTGCTCAGGACTCCATCTCT & NM_001315 \\
MAPKp38betaF & CCCGGACATATATCCAGTCC & AF001008 \\
MAPKp38betaR & TCACTGCTCAATCTCCAGG & AF001008 \\
MAPKp38gammaF & GCCCATCCCTACTTCGAGTC & U66243 \\
MAPKp38gammaR & CTTCACAGAGGCGTCTCCTT & U66243 \\
MAPKp38deltaF & GGCAGTTTAACGTGGCCTGTTA & AF092535 \\
MAPKp38deltaR & ACAGTGGATGAATGGAAGCAGC & AF092535 \\
\hline
\end{tabular}


a

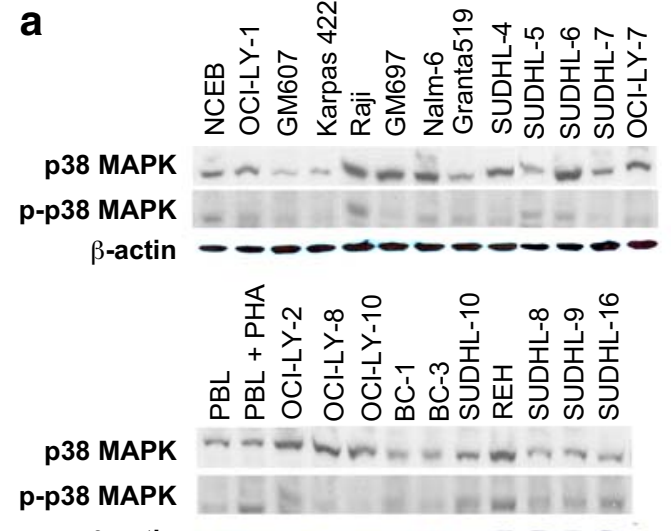

$\beta$-actin $-\infty-\infty-0-\infty-00$

b Reactive GC

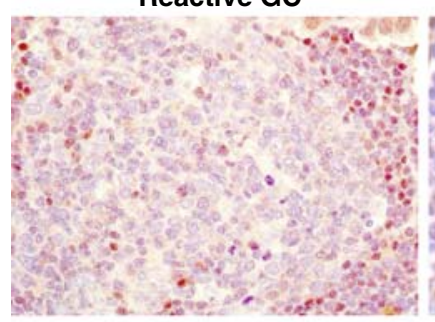

CLL/SLL high p-p38

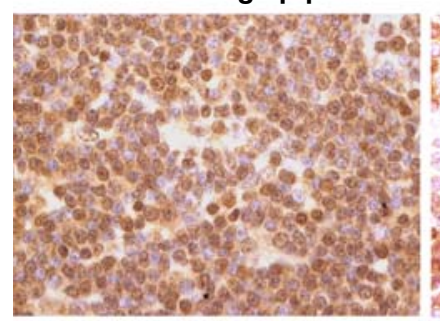

MCL high p-p38

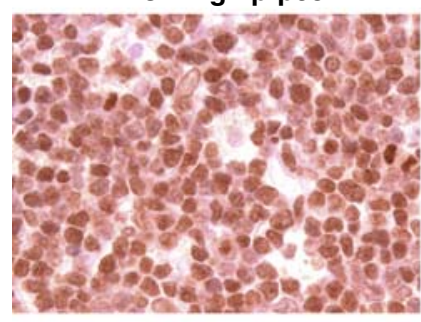

DLBCL high p-p38

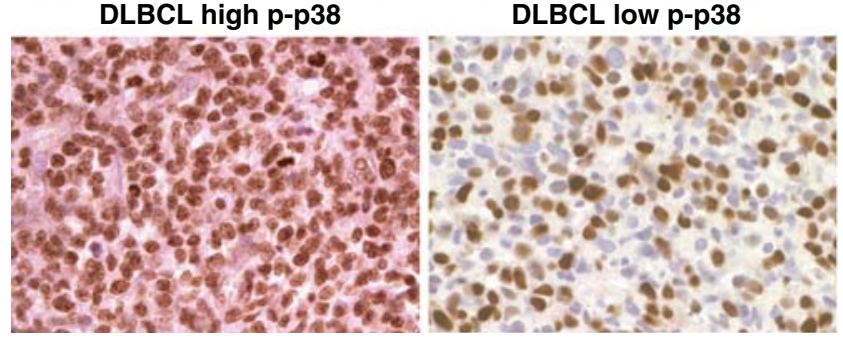

Expression of p38 MAPK and p-p38 MAPK

in DLBCL-derived cell lines

Using Western blot analysis, we evaluated the expression of p38 MAPK and p-p38 MAPK in five cell lines derived from DLBCLs, Sudhl-4, Sudhl-6, OCI-Ly1, OCI-Ly10, and
Fig. 1 a Western blot analyses of expression of native (p38 MAPK) and phosphorylated p38 MAPK (p-p38 MAPK) in human-derived B cell lymphoma cell lines. For comparison, p38 MAPK and p-p38 MAPK also done in peripheral blood lymphocytes (PBL) and phytohemagglutinin-stimulated $\mathrm{PBL}(\mathrm{PBL}+\mathrm{PHA})$. The experiments were performed in triplicate, and results shown are from the same experiment. b Immunohistochemical analysis of p-p38 MAPK in reactive lymphoid tissue and $\mathrm{B}$ cell NHL tissue samples. Germinal center B cells within reactive lymphoid tissue show negligible expression of p-p38 MAPK. Representative cases of FCL and CLL/ SLL demonstrating low p-p38 MAPK expression are shown. MCL and DLBCL demonstrating strong nuclear expression of p-p38 MAPK are shown

Sudhl-5. Both native p38 MAPK and p-p38 MAPK were detected in the five DLBCL cell lines (Fig. 2a, b). Except in Sudhl-6, the levels of both proteins in the DLBCL cell lines are relatively uniform, varying between 1 and 2.19 SDU for p38 MAPK and between 1 and 2.21 SDU for p-p38 MAPK, respectively (Fig. 2b). Interestingly, the levels of both p38 MAPK and p-p38 MAPK in Sudhl-6 are significantly higher than in the other four cell lines, with the levels of p38 MAPK and p-p38 MAPK being 8.01 and 3.72 SDU, respectively (Fig. 2b).

In vitro p38 MAPK activity in DLBCL-derived cell lines

To determine whether the expression of activated p38MAPK correlated with in vitro kinase activity, we used

a

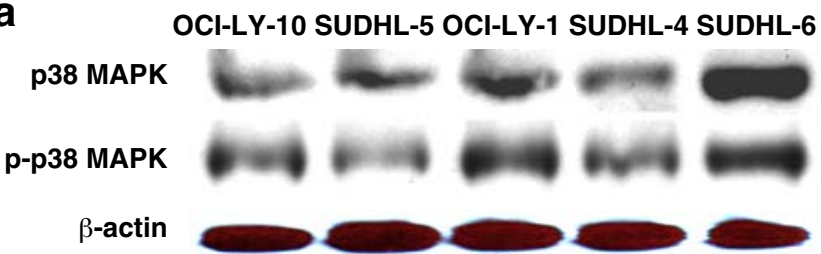

b

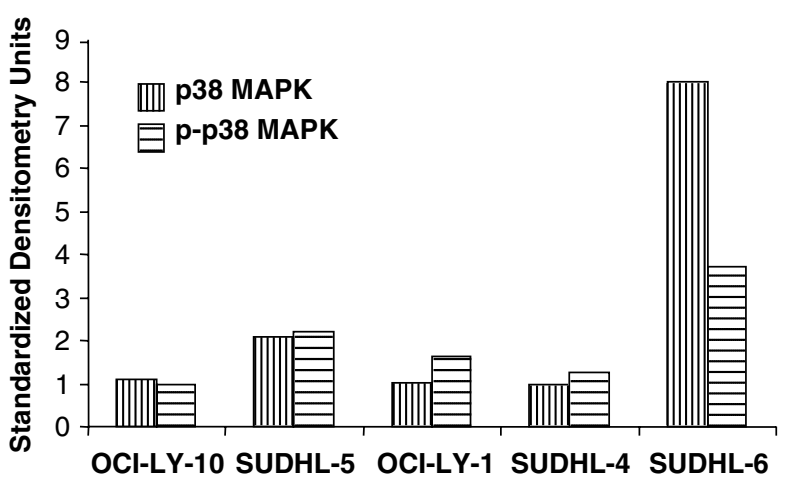

Fig. 2 a Representative Western blot analyses of p38 MAPK and p-p38 MAPK expression in five cell lines derived from DLBCLs, OCI-Ly-10, Sudhl-5, OCI-Ly-1, Sudhl-4, and Sudhl-6. b Relative expression of p38 and p-p38 MAPK in the five lymphoma-derived cell lines expressed in standardized densitometry units (SDU). Each data point represents the mean of triplicate assays 
an in vitro kinase assay to determine the activity of $\mathrm{p} 38$ MAPK. As shown in Fig. 3, the cell lysates from all five DLBCL-derived cell lines showed in vitro p38 MAPK activity as evidenced by the expression of phosphorylated ATF-2 which is a substrate of p38 MAPK. Sudhl-5 and Sudhl-6 cells display the highest level of p38 MAPK activity while Sudhl-4 cells express the lowest p38 MAPK activity. When the kinase activity of the cell lines is corrected to the level in Sudhl-4, the relatively p38 MAPK activity of Sudhl-5, Sudhl-6, Ly-1, and Ly-10 are 2.63, $2.41,1.86$, and 1.43 , respectively (Fig. 3b).

Effects of IL-4 on expression of p38 MAPK and p-p38 MAPK of DLBCL-derived cell lines

To determine whether IL-4, a key cytokine that regulates the differentiation and proliferation of normal Blymphocytes, regulates the expression of p38 MAPK and p-p38 MAPK in lymphoma-derived cell lines, we assessed the expression of these proteins after exposure to recombinant IL-4. Stimulation of the five cell lines to varying concentrations of IL-4 for $24 \mathrm{~h}$ showed no effect on the expression of native p38 MAPK in all cell lines (Figs. 4 and 5a). This was not the case for p-p38 MAPK. A dosedependent increase in p-p38 MAPK was seen in OCI-Ly-10 and Sudhl-5 cells; both are ABC-like DLBCL-derived cell lines, in contrast to GCB-like DLBCL-derived cell lines including OCI-Ly-1, Sudhl-4, and Sudhl-6 cells, where there was no significant effect on the levels of p-p38

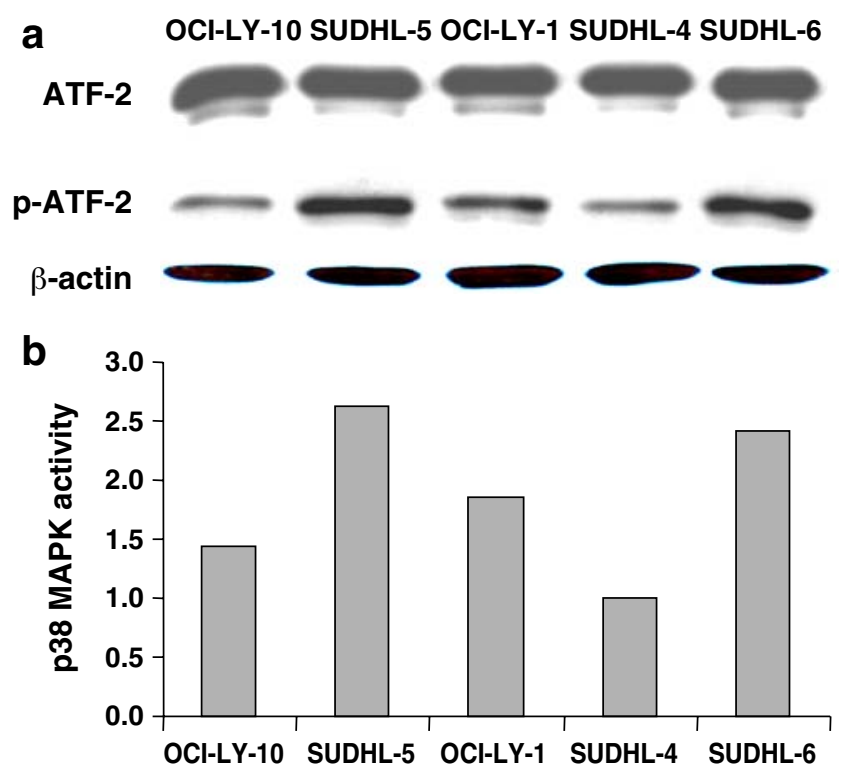

Fig. 3 a Representative Western blot analyses of p-ATF-2 and total ATF-2 proteins in five cell lines derived from DLBCLs, OCI-Ly-10, Sudhl-5, OCI-Ly-1, Sudhl-4, and Sudhl-6. b In vitro p38 MAPK activity in the five lymphoma-derived cell lines was determined by assessment of relative expression of p-ATF2 versus ATF-2. Each data point represents the mean of triplicate assays

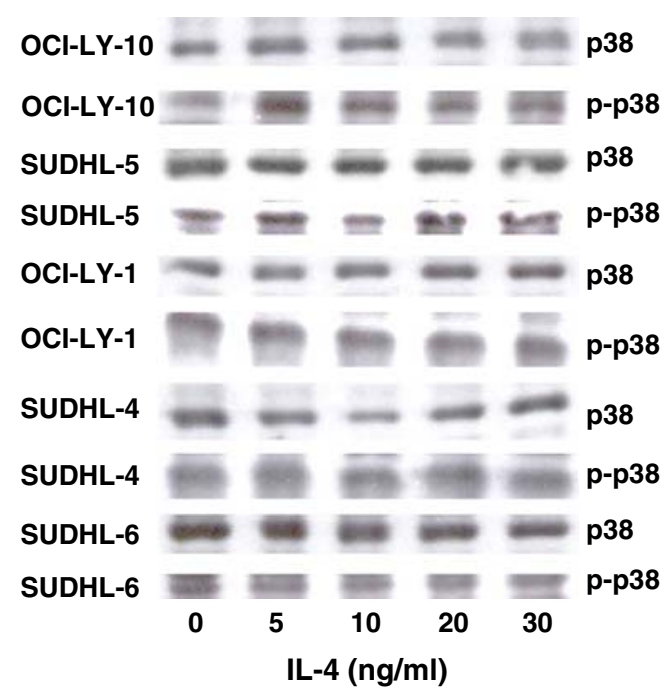

Fig. 4 Representative Western blot analyses of p38 MAPK and p-p38 MAPK proteins in lymphoma-derived cell lines exposed to varying concentrations of IL-4. The experiments were performed in triplicate and results shown are from the same experiment

MAPK (Figs. 4 and 5b). In OCI-Ly-10, the peak effective level of IL-4 was $5 \mathrm{ng} / \mathrm{ml}$ which resulted in a 2.3 -fold increase in p-p38 MAPK, while in Sudhl-5, the peak effective concentration of IL-4 was $10 \mathrm{ng} / \mathrm{ml}$ which resulted in a 2.6-fold increase in p-p38 MAPK. Higher concentrations of IL-4 demonstrated no significant increase in p-p38 MAPK expression in either OCI-Ly-10 or Sudhl-5 cell lines. These data indicate that, in cell lines that show relatively high constitutive expression of p38 MAPK and p-p38 MAPK (OCI-Ly-1, Sudhl-4, and Sudhl-6), IL-4 stimulation did not induce a significant increase in expression of the proteins. In cell lines that exhibited relatively lower levels of p38 MAPK and p-p38 MAPK (OCI-Ly-10 and Sudhl-5), however, IL-4 induced a significant increase in the expression of p-p38 MAPK but not native p38 MAPK.

Effect of IL-4 on in vitro p38MAPK activity in DLBCL-derived cell lines

We investigated the effect of IL-4 on the in vitro activity of p38 MAPK. IL-4 induced a dose-dependent increase in p38 MAPK in vitro activity in all the five cell lines (Fig. 6a, b). Except in Sudhl-5, the peak effective level of IL-4 is $5 \mathrm{ng} / \mathrm{ml}$ with the increase in p38 MAPK in vitro activity ranging from 1.57- to 2.33-fold of the basal levels. In these cell lines, further increase in IL-4 concentration was associated with a gradual decrease in p38 MAPK in vitro activity to the levels either compatible to the basal levels, as in Sudhl-6, OCI-Ly-1, and OCI-Ly-10, or below the basal level, as in Sudhl-4. In Sudhl-5, the peak effective level of IL-4 is $10 \mathrm{ng} / \mathrm{ml}$ which induced 3.21 times increase in p38 
a
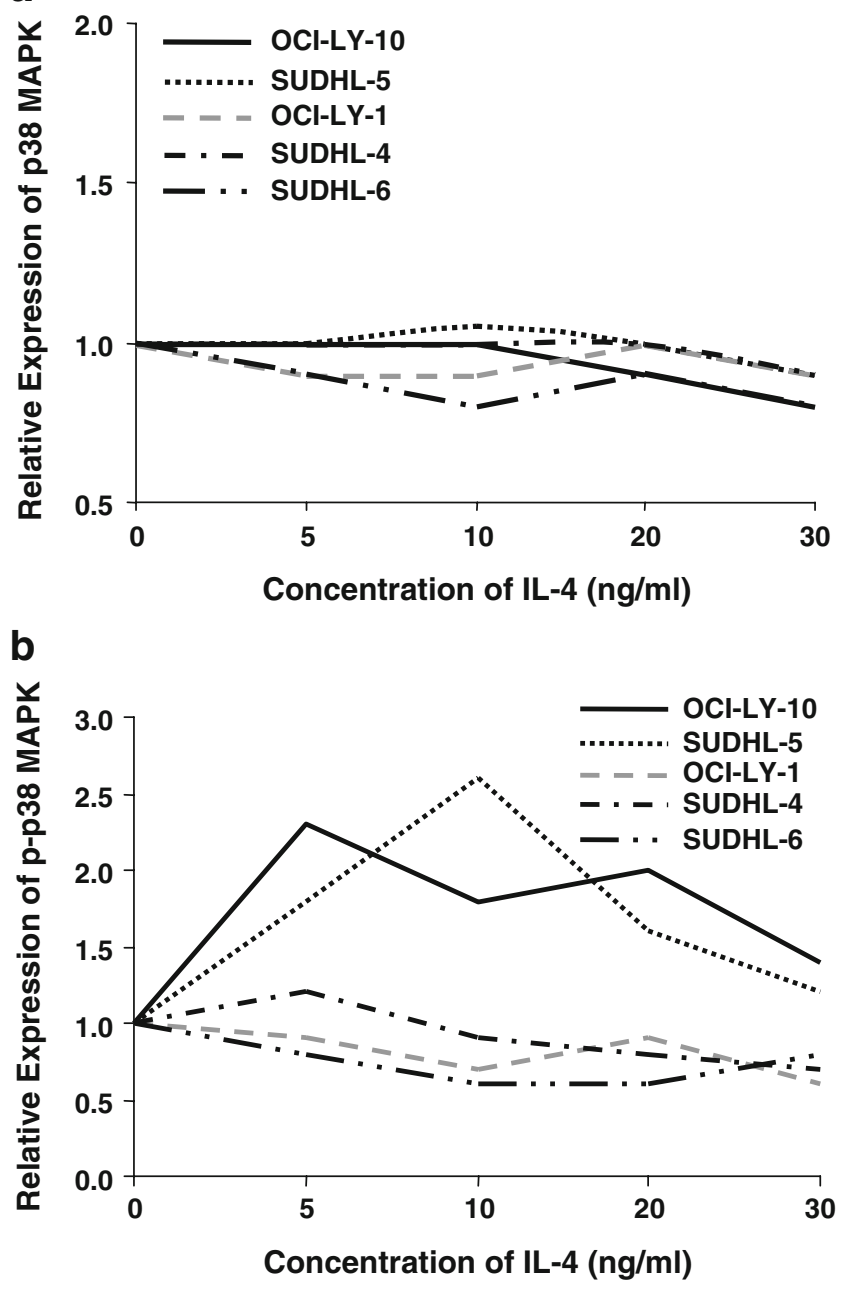

Fig. 5 a Graphic representation of effect of IL-4 on expression of p38 MAPK in B cell lymphoma-derived cell lines. Each data point represents the mean of triplicate assays and is corrected by the expression seen in the control untreated cells. b Graphic representation of effect of IL-4 on expression of p-p38 MAPK in lymphoma-derived cell lines. Each data point represents the mean of triplicate assays and is corrected by the expression seen in the control untreated cells

MAPK in vitro activity. The in vitro p38 MAPK activity in Sudhl-5 decreased with further increase in IL-4 concentration but did not return to basal level. Furthermore, the data indicate that there is a positive correlation between the levels of p-p38 MAPK and its in vitro kinase activity.

\section{Discussion}

Our previous studies showed increased expression of $\mathrm{p} 38$ MAPK transcript and protein in a subset of DLBCL arising from follicular lymphomas [26]. In addition, lymphoma cells in DLBCL displayed strong nuclear reactivity for p-p38 MAPK, while follicular lymphoma cells were often negative. Similar observations were obtained recently by
Ogasawara et al. [31] who found that freshly prepared cells from B cell lymphomas showed constitutive in vitro activity of p38 MAPK and ERK but not Ras, Akt kinase, and JNK kinase. These observations suggest that constitutive activation of the p38 MAPK pathway may be implicated in lymphomagenesis.

In the current study, we have examined the expression of p38 and p-p38 MAPK proteins in cell lines derived from a variety of B cell-derived NHL. As shown in Fig. 1, both proteins were detected in all human B cell lymphoma cell lines. As compared with normal lymphocytes isolated from peripheral blood, most of the cell lines derived from B cell neoplasms show up-regulated expression of p38 MAPK. The expression of p-p38 MAPK, however, was lower and more variable. These data suggest that the p38 MAPK is expressed and activated in neoplastic cells derived from B cell neoplasms. Immunohistochemistry demonstrated that, in reactive lymph nodes and tonsils, there were negligible
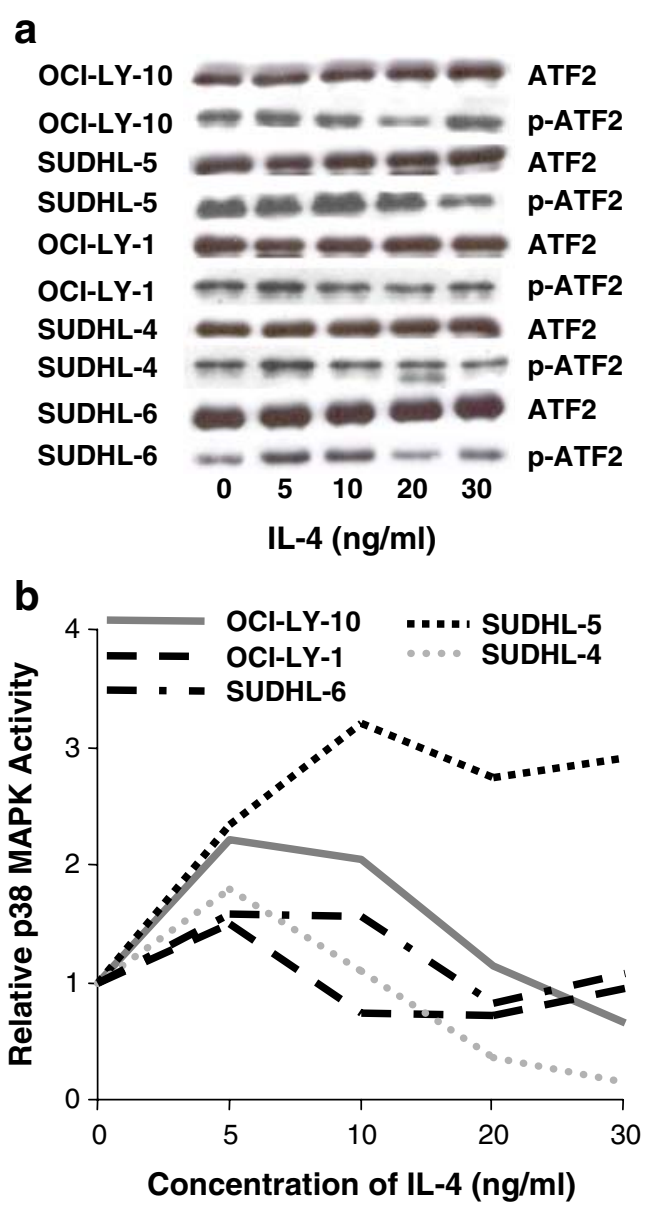

Fig. 6 a Effect of IL-4 on in vitro p38 MAPK activity in lymphomaderived cell lines. The expression of p-ATF-2 which serves as a substrate of $\mathrm{p} 38$ MAPK activity is measured relative to that of total ATF-2. b Graphic representation of relative in vitro p38 MAPK activity detected by increasing concentration of IL-4. Each data point represents the mean of three replicate assays and is corrected by the level seen in the control untreated cells 
levels of p-p38 MAPK in germinal center B cells, while the mantle B cells showed weak cytoplasmic expression. Analysis of primary tissues of B cell NHLs also demonstrated constitutive expression of activated and nuclear p-p38MAPK in a subset of lymphomas while germinal center B cells showed negligible levels. Interestingly, the low-grade B cell lymphomas (CLL/SLL and FCL) demonstrated lower percentage of tumor cells exhibiting p-p38 MAPK expression, while the aggressive lymphomas (MCL and DLBCL) exhibited higher percentage of cases and tumor cells exhibiting p-p38 MAPK expression. The p-p38 expression does not merely indicate proliferation status since p-p38 MAPK is not expressed in reactive germinal center B cells (Fig. 1b). The high percentage of MCL and DLBCL cases with p-p38 MAPK expression $(78 \%$ and $93 \%$, respectively) may be reflective of the presence of cytokines or growth factors that activate the p38 MAPK pathway in these tumors.

We determined whether expression of $\mathrm{p} 38$ MAPK and p-p38 MAPK is associated with its functional kinase activity. As demonstrated in Fig. 3a, b, cell lysates from five DLBCL-derived cell lines showed readily detectable in vitro p38 MAPK activity using ATF2, a natural substrate for the kinase activity of $\mathrm{p} 38$ MAPK. There was a positive correlation between the levels of p-p38 MAPK as determined by Western blot analysis and the in vitro p38 MAPK activity.

Although the role of p38 MAPK is thought to be central to many key cellular processes such as the production of IL-6, cell proliferation, and apoptosis in many cell systems, the regulation of p38 MAPK expression in normal and neoplastic lymphocytes has not received much attention in the literature. CD40 ligand provides a co-stimulatory signal that induces p38 MAPK activation and results in the differentiation and proliferation of B cells [25]. Type I interferons can activate p38 MAPK in T-lymphoblastic leukemia-derived cells and Burkitt lymphoma-derived cells [32, 33], and toll-like receptor 2 can induce p38 MAPK expression in HEK293 cells [34]. In addition, recent studies found that IL-4 induces the activation of p38 MAPK in endometriotic stromal cells [35].

However, little is known regarding the extracellular regulators of $\mathrm{p} 38$ MAPK expression in neoplastic B cells. Thus, we investigated the potential role of IL-4 in the regulation of the expression and activity of p38 MAPK. IL4 is a well characterized cytokine that is produced by normal CD4 $+\mathrm{T}$ cells and is important in regulation of normal B cell differentiation and proliferation [36]. High levels of IL-4 have been observed in culture supernatants from $\mathrm{B}$ and $\mathrm{T}$ cells from chronic lymphocytic leukemia (CLL) patients $[37,38]$. IL-4 may promote survival and viability of CLL cells by preventing apoptosis [37]. Our current study data shows that IL-4 stimulation induced the expression of p-p38 MAPK as well as the in vitro p38 MAPK activity in cell lines derived from B cell lymphomas while previous studies showed that IL-4 failed to stimulate the enzymatic activity of $\mathrm{p} 38$ MAPK or MAPKAP kinase2 , a downstream kinase in the p38 MAPK pathway. These studies were, however, limited to the analysis of primary mast cell cultures and cultured factor-dependent hematopoietic cell lines MC/9 and DS-MACII [4]. The finding that the level of p-p38 MAPK in OCI-LY-10 cell line reached a plateau at $5 \mathrm{ng} / \mathrm{ml} \mathrm{Il-4}$ with minimal increase at $20 \mathrm{ng} / \mathrm{ml}$ Il-4 may be due to the susceptibility of OCI-LY-10 to low-dose Il-4 sensitization or prolonged receptor-ligand interactions. These observations further suggest that IL-4mediated activation of p38 MAPK may be highly cell-type specific. IL-4 has been shown to activate p38 MAPKdependent pathways in a number of other cell types including keratinocytes [39].

We also found that expression of p-p38 MAPK and in vitro kinase activity among cell lines derived from DLBCLs was cell-type dependent. Whereas expression and activity of p38MAPK in Sudhl-4, Sudhl-6, and OCILy-1 cells were higher than in Sudhl-5 and OCI-Ly-10 cells, the latter cell lines responded more potently to induction by IL-4. Interestingly, these two cell lines do not harbor the $t(14 ; 18)$ chromosomal aberration and have been regarded as equivalents of the activated $\mathrm{B}$ cell lymphoma subtype in cDNA microarray studies [40].

Tissue distribution of different isoforms of p38 MAPK mRNA has been studied [2, 13-18, 41-43]; however, there is no information on expression of these isoforms in B cells or B cell lymphoma cells. Our data show that both reactive germinal center $\mathrm{B}$ cells and cells derived from DLBCL express all four isoforms of $\mathrm{p} 38$ MAPK at the RNA level (supplementary Fig. 1). The most predominant isoform of p38 MAPK expressed in cell lines derived from DLBCL was $\mathrm{p} 38 \gamma$, followed by $\mathrm{p} 38 \beta$, while the expression of $\mathrm{p} 38 \alpha$ and $\mathrm{p} 38 \delta$ was minimal. These data further highlight the complexity of regulation of $\mathrm{p} 38$ MAPK isoform expression by which IL-4 may play a role in regulating p38 MAPK pathway (supplementary Fig. 2).

In conclusion, our data indicate that constitutive activation of the p38 MAPK may represent an important pathogenetic mechanism in B cell lymphomas. This study documents the differential expression of isoforms of the p38 MAPK gene in DLBCL-derived cell lines, implicating its possible deregulation in the pathogenesis of these neoplasms. We present, evidence that IL-4 may be an important regulator of the p38 MAPK signaling pathway with stimulatory effects on gene expression, protein levels, and in vitro kinase activity. The data suggest a possible role for IL-4-mediated activation of p38 MAPK signaling pathway in the pathogenesis of B cell lymphomas. 
The authors have no affiliations or involvement in any organization with a direct financial interest in the subject matter or materials discussed in the manuscript.

\section{References}

1. Han J, Lee JD, Bibbs L, Ulevitch RJ (1994) A MAP kinase targeted by endotoxin and hyperosmolarity in mammalian cells. Science 265:808-811

2. Lee JC, Laydon JT, McDonnell PC, Gallagher TF, Kumar S, Green D, McNulty D, Blumenthal MJ, Heys JR, Landvatter SW et al (1994) A protein kinase involved in the regulation of inflammatory cytokine biosynthesis. Nature 372:739-746

3. Rouse J, Cohen P, Trigon S, Morange M, Alonso-Llamazares A, Zamanillo D, Hunt T, Nebreda AR (1994) A novel kinase cascade triggered by stress and heat shock that stimulates MAPKAP kinase- 2 and phosphorylation of the small heat shock proteins. Cell 78:1027-1037

4. Foltz IN, Lee JC, Young PR, Schrader JW (1997) Hemopoietic growth factors with the exception of interleukin-4 activate the p38 mitogen-activated protein kinase pathway. J Biol Chem 272:3296-3301

5. Kramer RM, Roberts EF, Strifler BA, Johnstone EM (1995) Thrombin induces activation of p38 MAP kinase in human platelets. J Biol Chem 270:27395-27398

6. Raingeaud J, Whitmarsh AJ, Barrett T, Derijard B, Davis RJ (1996) MKK3- and MKK6-regulated gene expression is mediated by the p38 mitogen-activated protein kinase signal transduction pathway. Mol Cell Biol 16:1247-1255

7. Han J, Jiang Y, Li Z, Kravchenko VV, Ulevitch RJ (1997) Activation of the transcription factor MEF2C by the MAP kinase p38 in inflammation. Nature 386:296-299

8. Shapiro L, Dinarello CA (1995) Osmotic regulation of cytokine synthesis in vitro. Proc Natl Acad Sci U S A 92:12230-12234

9. Miyazawa K, Mori A, Miyata H, Akahane M, Ajisawa Y, Okudaira H (1998) Regulation of interleukin-1beta-induced interleukin-6 gene expression in human fibroblast-like synoviocytes by p38 mitogen-activated protein kinase. J Biol Chem 273:24832-24838

10. Xia Z, Dickens M, Raingeaud J, Davis RJ, Greenberg ME (1995) Opposing effects of ERK and JNK-p38 MAP kinases on apoptosis. Science 270:1326-1331

11. Ichijo H, Nishida E, Irie K, ten Dijke P, Saitoh M, Moriguchi T, Takagi M, Matsumoto K, Miyazono K, Gotoh Y (1997) Induction of apoptosis by ASK1, a mammalian MAPKKK that activates SAPK/JNK and p38 signaling pathways. Science 275:90-94

12. Kummer JL, Rao PK, Heidenreich KA (1997) Apoptosis induced by withdrawal of trophic factors is mediated by p38 mitogenactivated protein kinase. J Biol Chem 272:20490-20494

13. Jiang Y, Chen C, Li Z, Guo W, Gegner JA, Lin S, Han J (1996) Characterization of the structure and function of a new mitogenactivated protein kinase (p38beta). J Biol Chem 271:17920-17926

14. Stein B, Yang MX, Young DB, Janknecht R, Hunter T, Murray BW, Barbosa MS (1997) p38-2, a novel mitogen-activated protein kinase with distinct properties. J Biol Chem 272:19509-19517

15. Lechner C, Zahalka MA, Giot JF, Moller NP, Ullrich A (1996) ERK6, a mitogen-activated protein kinase involved in $\mathrm{C} 2 \mathrm{C} 12$ myoblast differentiation. Proc Natl Acad Sci U S A 93:4355-4359

16. Li Z, Jiang Y, Ulevitch RJ, Han J (1996) The primary structure of p38 gamma: a new member of p38 group of MAP kinases. Biochem Biophys Res Commun 228:334-340

17. Wang XS, Diener K, Manthey CL, Wang S, Rosenzweig B, Bray J, Delaney J, Cole CN, Chan-Hui PY, Mantlo N, Lichenstein HS,
Zukowski M, Yao Z (1997) Molecular cloning and characterization of a novel p38 mitogen-activated protein kinase. J Biol Chem 272:23668-23674

18. Jiang Y, Gram H, Zhao M, New L, Gu J, Feng L, Di Padova F, Ulevitch RJ, Han J (1997) Characterization of the structure and function of the fourth member of p38 group mitogen-activated protein kinases, p38delta. J Biol Chem 272:30122-30128

19. Lee JC, Kassis S, Kumar S, Badger A, Adams JL (1999) p38 mitogen-activated protein kinase inhibitors-mechanisms and therapeutic potentials. Pharmacol Ther 82:389-397

20. Cohen P (1997) The search for physiological substrates of MAP and SAP kinases in mammalian cells. Trends Cell Biol 7:353-361

21. Wilk-Blaszczak MA, Stein B, Xu S, Barbosa MS, Cobb MH, Belardetti F (1998) The mitogen-activated protein kinase p38-2 is necessary for the inhibition of N-type calcium current by bradykinin. J Neurosci 18:112-118

22. Conrad PW, Rust RT, Han J, Millhorn DE, Beitner-Johnson D (1999) Selective activation of p38alpha and p38gamma by hypoxia. Role in regulation of cyclin D1 by hypoxia in PC12 cells. J Biol Chem 274:23570-23576

23. Nemoto S, Xiang J, Huang S, Lin A (1998) Induction of apoptosis by SB202190 through inhibition of p38beta mitogen-activated protein kinase. J Biol Chem 273:16415-16420

24. Enslen H, Brancho DM, Davis RJ (2000) Molecular determinants that mediate selective activation of p38 MAP kinase isoforms. Embo J 19:1301-1311

25. Craxton A, Shu G, Graves JD, Saklatvala J, Krebs EG, Clark EA (1998) p38 MAPK is required for CD40-induced gene expression and proliferation in B lymphocytes. J Immunol 161:3225-3236

26. Elenitoba-Johnson KS, Jenson SD, Abbott RT, Palais RA, Bohling SD, Lin Z, Tripp S, Shami PJ, Wang LY, Coupland RW, Buckstein R, Perez-Ordonez B, Perkins SL, Dube ID, Lim MS (2003) Involvement of multiple signaling pathways in follicular lymphoma transformation: p38-mitogen-activated protein kinase as a target for therapy. Proc Natl Acad Sci U S A 100: 7259-7264

27. Drexler HG (2001) The leukemia-lymphoma cell line factsbook. Academic Press, Boston, pp 263-266

28. Morgan JW, Kouttab N, Ford D, Maizel AL (2000) Vitamin Dmediated gene regulation in phenotypically defined human B cell subpopulations. Endocrinology 141:3225-3234

29. Morrison TB, Weis JJ, Wittwer CT (1998) Quantification of lowcopy transcripts by continuous SYBR Green I monitoring during amplification. Biotechniques 24:954-958 960, 962

30. Elenitoba-Johnson KS, Bohling SD, Jenson SD, Lin Z, Monnin KA, Lim MS (2002) Fluorescence PCR quantification of cyclin D1 expression. J Mol Diagnostics 4:90-96

31. Ogasawara T, Yasuyama M, Kawauchi K (2003) Constitutive activation of extracellular signal-regulated kinase and p38 mitogen-activated protein kinase in B-cell lymphoproliferative disorders. Int J Hematol 77:364-370

32. Lin Z, Crockett DK, Jenson SD, Lim MS, Elenitoba-Johnson KS (2004) Quantitative proteomic and transcriptional analysis of the response to the p38 MAPK inhibitor SB203580 in transformed follicular lymphoma cells. Mol Cell Proteomics 3:820-833

33. Uddin S, Majchrzak B, Woodson J, Arunkumar P, Alsayed Y, Pine R, Young PR, Fish EN, Platanias LC (1999) Activation of the p38 mitogen-activated protein kinase by type I interferons. J Biol Chem 274:30127-30131

34. Vasselon T, Hanlon WA, Wright SD, Detmers PA (2002) Toll-like receptor 2 (TLR2) mediates activation of stress-activated MAP kinase p38. J Leukoc Biol 71:503-510

35. Yang ZO, Hirota Y, Osuga Y, Hamasaki K, Hasegawa A, Tajima T, Hirata T, Koga K, Yoshino O, Harada M, Takemura Y, Nose E, Yano T, Taketani Y (2008) Interleukin-4 stimulates proliferation of endometriotic stromal cells. Am J Pathol 173:463-469 
36. Kay NE, Pittner BT (2003) IL-4 biology: impact on normal and leukemic CLL B cells. Leuk Lymphoma 44:897-903

37. Mainou-Fowler T, Proctor SJ, Miller S, Dichnsos AM (2001) Expression and production of interleukin 4 in B-cell chronic lymphocytic leukaemia. Leuk Lymphoma 42:689-698

38. Kay NE, Han L, Bone N, Williams G (2001) Interleukin 4 content in chronic lymphocytic leukaemia (CLL) B cells and blood CD8+ T cells from B-CLL patients: impact on clonal B-cell apoptosis. Br J Haematol 112:760-767

39. Wery-Zennaro S, Zugaza JL, Letourneur M, Bertoglio J, Pierre J (2000) IL-4 regulation of IL-6 production involves Rac/Cdc42and p38 MAPK-dependent pathways in keratinocytes. Oncogene 19:1596-1604

40. Alizadeh AA, Eisen MB, Davis RE, Ma C, Lossos IS, Rosenwald A, Boldrick JC, Sabet H, Tran T, Yu X, Powell JI, Yang L, Marti GE, Moore T, Hudson J Jr, Lu L, Lewis DB, Tibshirani R, Sherlock G, Chan WC, Greiner TC, Weisenburger DD, Armitage
JO, Warnke R, Levy R, Wilson W, Grever MR, Byrd JC, Botstein D, Brown PO, Staudt LM (2000) Distinct types of diffuse large Bcell lymphoma identified by gene expression profiling. Nature 403:503-511

41. Enslen H, Raingeaud J, Davis RJ (1998) Selective activation of p38 mitogen-activated protein (MAP) kinase isoforms by the MAP kinase kinases MKK3 and MKK6. J Biol Chem 273:17411748

42. Kumar S, McDonnell PC, Gum RJ, Hand AT, Lee JC, Young PR (1997) Novel homologues of CSBP/p38 MAP kinase: activation, substrate specificity and sensitivity to inhibition by pyridinyl imidazoles. Biochem Biophys Res Commun 235: 533-538

43. Hale KK, Trollinger D, Rihanek M, Manthey CL (1999) Differential expression and activation of p38 mitogen-activated protein kinase alpha, beta, gamma, and delta in inflammatory cell lineages. J Immunol 162:4246-4256 